\title{
INVESTIGACIONES
}

\section{Revisión sistemática del aula invertida en el Ecuador: aproximación al estado del arte}

\author{
Systematic review of the flipped classroom in Ecuador: \\ approach to the state of the art \\ Revisão sistemática da sala de aula invertida no Equador: \\ uma abordagem ao estado da arte
}

\author{
Abigail Alejandra Cantuña Avila ${ }^{a}$, Carolina Elizabeth Cañar Tapia ${ }^{a}$ \\ ${ }^{a}$ Universidad Politécnica Salesiana, Ecuador. \\ acantunaa@gmail.com,ct674437@gmail.com
}

\begin{abstract}
RESUMEN
La educación ha evolucionado a lo largo del tiempo con nuevas estrategias y metodologías integrando las TIC -Tecnologías de la Información y Comunicación- con el fin de cambiar las prácticas tradicionales. Por esta razón se ha considerado al aula invertida como un modelo innovador que fusiona el espacio de aprendizaje en casa con las clases presenciales; despertado la curiosidad y la participación de los estudiantes, transformando las experiencias en el salón de clase. Este artículo tiene como objetivo presentar una revisión sistemática construyendo un estado del arte sobre el aula invertida en el Ecuador, utilizando una guía de ocho pasos propuesta por Okoli y Schabram (2010), las directrices PRISMA y la técnica de análisis documental, cuyo propósito es garantizar la rigurosidad, transparencia y claridad de la investigación; brindando un punto de partida hacía futuras investigaciones. Se consultó las bases de datos Scielo, Redalyc y se incluye el buscador Google Académico durante el período de 2017 a agosto de 2020. Entre los resultados, se destaca que el aula invertida se ha aplicado en todas las asignaturas, con mayor incidencia en el aprendizaje del idioma inglés, debido al desinterés del estudiante y dificultades para desarrollar las cuatro habilidades lingüísticas que implica aprender un nuevo idioma. En definitiva, es importante impulsar más estudios sobre esta metodología, ya que presenta excelentes resultados, pero aún no existen evidencias durante la pandemia.

Palabras claves: Aula invertida, educación, aprendizaje significativo, TIC, estrategia de aprendizaje.
\end{abstract}

\begin{abstract}
Education has envolved over time with new strategies and methodologies integrating the ICT-Information and communication technologies- with the final of changing the traditional practices. For this reason, the flipped classroom has been considered an innovative model that merges the learning space at home with face to face classes; awakening the curiosity and participation of students, changing the experience in the classroom. This article has the objective present a systematic review building a state of the art on the flipped classroom in Ecuador, using an eight step guide proposed by Okoli y Schabram (2010), the PRISMA guidelines and the technique of documental analysis, whose porpouse is to guarantee the rigor, transparency and clarity of the investigation; providing a starting point for future research. The Scielo and Redalyc databases were consulted and the Google Scholar search engine was included during the period from 2017 to August 2020. Among the results, it is emphasized that the flipped classroom has been applied in all the subjects, with more incidence in the learning of the English language. Due to the disinterest of students and difficult to develop the four linguistic ability that implied learn a new language. Definitely, it is important to promote more studies about this methodology since it presents excellent results, but during the pandemic, it hasn't existed any evidence yet.
\end{abstract}

Key words: Flipped classroom, education, meaningful learning, ICT, learning strategy. 


\section{RESUMO}

A educação tem evoluído ao longo do tempo com novas estratégias e metodologias integrando as TIC -Tecnologias de Informação e Comunicação- de forma a mudar as práticas tradicionais. Por isso, a sala de aula invertida tem sido considerada um modelo inovador que mescla o espaço de aprendizagem em casa com as aulas presenciais; despertou a curiosidade e participação dos alunos, transformando as vivências em sala de aula. Este artigo tem como objetivo apresentar uma revisão sistemática construindo um estado da arte sobre a sala de aula invertida no Equador, utilizando um guia de oito etapas proposto por Okoli y Schabram (2010), as diretrizes do PRISMA e a técnica de análise de documentos, cujo objetivo é garantir o rigor, transparência e clareza da investigação; fornecendo um ponto de partida para pesquisas futuras. Foram consultadas as bases de dados Scielo e Redalyc e incluído o buscador Google Academic durante o período de 2017 a agosto de 2020. Dentre os resultados, destacase que a aula invertida foi aplicada em todas as disciplinas, com maior impacto na aprendizagem da Língua Inglesa, devido ao desinteresse do aluno e às dificuldades em desenvolver as quatro competências linguísticas que a aprendizagem de uma nova língua implica. Em suma, é importante promover mais estudos sobre essa metodologia, pois ela apresenta excelentes resultados, mas ainda não há evidências durante a pandemia.

Palavras-chave: Sala de aula invertida, educação, aprendizagem significativa, TIC, estratégia de aprendizagem.

\section{INTRODUCCIÓN}

La educación ha ido evolucionando a lo largo del tiempo mejorando teorías, modelos pedagógicos, metodologías, técnicas e incluso incorporando recursos tecnológicos. Un claro ejemplo de esto es la metodología activa conocida como aula invertida o en inglés flipped classroom. La misma que inicia, con el pensamiento de los pioneros estadunidense Jonathan Bergmann y Aaron Sams. Estos docentes al detectar que por diversos motivos los estudiantes no podían asistir a clases; surge la iniciativa de proporcionarles videos tutoriales para que ellos observen desde sus hogares y en la siguiente clase se trabaje con actividades que refuercen dicha temática.

El aula invertida se define como un método de aprendizaje cuyo propósito es que el alumno asuma un rol mucho más activo para estudiar cada tema a través de videos, foros, diálogos, entre otros y así lograr desarrollar un proceso de aprendizaje significativo (Salazar, 2017). Por esta razón, esta metodología promueve el protagonismo del educando utilizando herramientas tecnológicas con la finalidad de facilitar el proceso de enseñanzaaprendizaje y aprovechar el tiempo en el aula para aclarar dudas.

En la actualidad los procesos de formación académica requieren principalmente de acceso a internet como medio de comunicación, donde los programas o páginas web son informativos y educativos. "En el aula invertida se otorga a los estudiantes la responsabilidad de consultar los contenidos de la clase en sus hogares, de esta manera pueden resolver sus dudas y trabajar los conceptos en clases de forma individual" (Bermeo y Zorrilla, 2019, p. 19). De esta manera, el docente analiza la mejor manera para transmitir conocimientos a partir de herramientas tecnológicas para conceptualizar el contenido del estudiante desde el hogar y que se desarrollen actividades y tareas en el aula que permitan trabajar y desenvolverse en el proceso de información.

Además, está metodología se caracteriza por ser ubicua, es decir se puede aprender en cualquier lugar y momento. En este caso, es importante la intervención del docente para planificar las experiencias de aprendizaje tanto para el aula como el hogar. Parra ello, debe considerar el ritmo de aprendizaje del estudiante y el tiempo adecuado que este dedicará para desarrollar conocimientos previos.

En este artículo se entiende como aula invertida a la metodología que tiene como meta innovar e invertir los procesos educativos. Esta propone, un primer momento desde casa, 
en donde el estudiante investigue e interaccione con el contenido digital que el docente le proporcione -videos, podcast, actividades en entornos virtuales, entre otros-; para que en la clase este participe activamente en actividades que le permitan: poner en práctica su conocimiento y resolver dudas e inquietudes. En otras palabras, el docente optimiza el tiempo en el aula para retroalimentar y reforzar el aprendizaje mediante proyectos, debates, experimentos, etc. El aula invertida en el Ecuador tiene los siguientes enfoques teóricos: b-learning o modelo de aprendizaje hibrido, taxonomía de Bloom, y aprendizaje significativo. A continuación, se conceptualizan cada uno de ellos.

El b-learning o mejor conocido como aprendizaje híbrido, “integra el modo en línea y cara a cara, para crear una experiencia de aprendizaje integrada, proporcionando a los estudiantes una mayor flexibilidad, apoyo y nuevos canales de comunicación entre profesores y estudiantes" (Mejía et al., 2017, p. 353). En otras palabras, este modelo de aprendizaje utiliza los recursos TIC, entornos virtuales y plataformas para generar procesos educativos que potencien la investigación, el trabajo colaborativo e individual.

El ciclo de aprendizaje en el aula invertida se basa en la Taxonomía de Bloom; donde se desarrollan las actividades asincrónicas para formar nuevos significados mediante las destrezas de recordar, entender y aplicar. Por otra parte, la acciones sincrónicas en el aula responden a los niveles: analizar, evaluar y crear, es decir, el docente se encarga de proponer actividades como la experimentación, demostración y aplicación de conocimientos ya adquiridos (Cedeño y Vigueras, 2020).

El aprendizaje significativo se entiende como procesos cognitivos que permiten relacionar los aprendizajes nuevos con los ya adquiridos (Ortega, 2017). Esto permite que los estudiantes no solo memoricen conceptos y situaciones, sino que comprender dicha información. Por lo que, el docente debe planificar actividades que se relacionen con el contexto del educando.

\section{METODOLOGÍA}

La revisión sistemática de literatura (RSL) es un proceso arduo que identifica, evalúa e interpreta toda la información relevante sobre una pregunta de investigación, área temática o fenómeno de interés (Kitchenham, 2004); esta ofrece un punto de partida para la comunidad académica y necesita de planificación, búsqueda, selección y sistematización.

El presente estudio utiliza la metodología propuesta por Okoli y Schabram (2010) la misma que plantea una guía de ocho pasos: identificar el objetivo de la revisión; protocolo y formación; búsqueda de literatura; selección; evaluación de la calidad; extracción de datos; síntesis del estudio y redactar la reseña. A continuación, se exponen las preguntas que delimitaron la investigación: a) ¿Cuál es el estado de investigación científica sobre el aula invertida en el Ecuador? y b) ¿Qué resultados se han generado con la implementación del aula invertida?

El período de búsqueda se estableció a 4 años, es decir publicaciones entre enero de 2017 y agosto de 2020, considerando el notable crecimiento e interés hacia nuevas estrategias que cambien las prácticas tradicionales, considerando al aula invertida como una alternativa. Para ello, se utilizó las bases de datos especializadas en educación Scielo, Redalyc y el buscador académico Google Scholar. Las palabras clave que guiaron la revisión fueron "aula invertida" y "flipped classroom" ambas delimitadas en Ecuador, donde se centra el estudio. Se visualizan el número de resultados por cada fuente en la tabla 1. 
Tabla 1. Resultados por descriptor delimitando en Ecuador

\begin{tabular}{|l|l|c|}
\hline \multicolumn{1}{|c|}{ Fuente } & \multicolumn{1}{c|}{ Descriptor } & Número de resultados \\
\hline \multirow{2}{*}{$\begin{array}{l}\text { SciELO } \\
\text { https://scielo.org/es/ }\end{array}$} & aula invertida & 3 \\
\cline { 2 - 3 } $\begin{array}{l}\text { Redalyc } \\
\text { https://www.redalyc.org/ }\end{array}$ & flipped classroom & 1 \\
\hline \multirow{2}{*}{$\begin{array}{l}\text { Google Scholar } \\
\text { https://scholar.google.com/schhp?hl=es }\end{array}$} & aula invertida & 1 \\
\cline { 2 - 3 } & flipped classroom & 1130 \\
\hline Total & aula invertida & 1260 \\
\hline
\end{tabular}

Fuente: elaboración propia.

Los criterios de inclusión y exclusión que se establecieron para la indagación fueron: trabajos publicados entre 2017 y agosto de 2020, documentos en español, área temática: educación, disponibilidad del texto completo y estudios cuantitativos, cualitativos o mixtos. Este proceso permitió depurar la información, descartando los documentos que no contribuyen con información suficiente relacionada con el objetivo de la investigación. La figura 1, muestra el diagrama de flujo del proceso de búsqueda y selección siguiendo las directrices PRISMA y cuyo propósito fue garantizar su transparencia y claridad.

Figura 1. Diagrama de flujo PRISMA
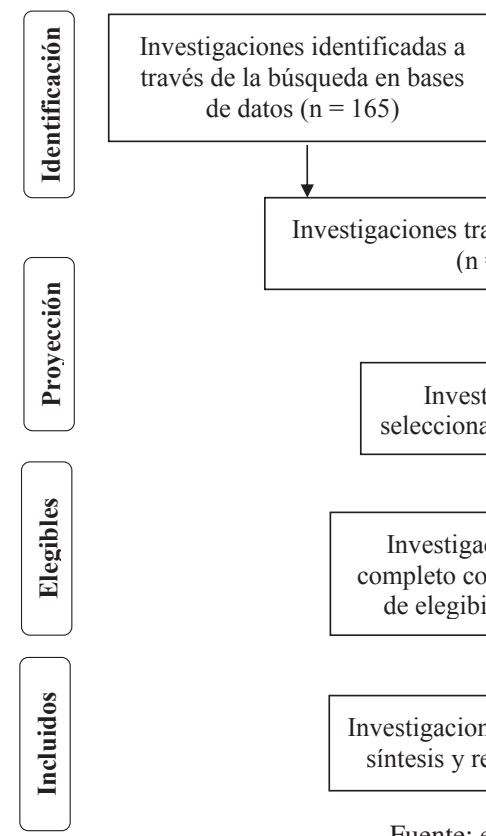

$$
(\mathrm{n}=155)
$$
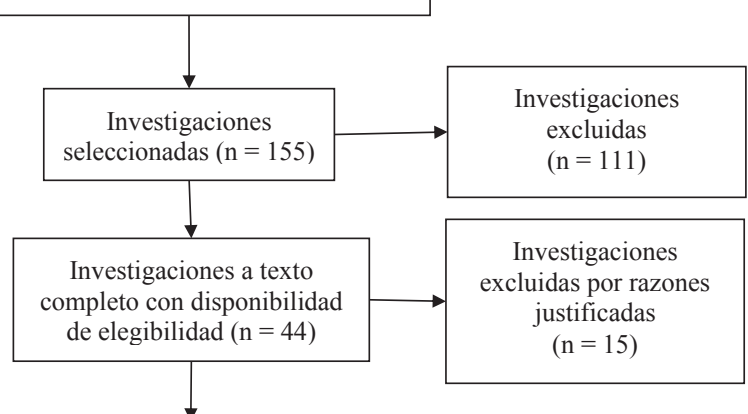

Investigaciones incluidas para síntesis y revisión $(\mathrm{n}=29)$

Fuente: elaboración propia. 


\section{RESULTADOS Y DISCUSIÓN}

A partir de las interrogantes planteadas anteriormente se presentan los resultados de la investigación tanto cuantitativos como cualitativos, en el orden mencionado. Para esto, se utilizó una matriz recopilando la información esencial de los 29 documentos seleccionados.

\section{1. ¿CUÁL ES EL ESTADO DE INVESTIGACIÓN CIENTÍFICA SOBRE EL AULA INVERTIDA EN EL} ECUADOR?

Esta interrogante surge debido a que la temática: aula invertida en el Ecuador es de gran interés para los docentes que buscan integrar nuevos metodos de aprendizaje ante la realidad educativa que se enfrentan debido a la pandemia; pues esta metodología denominada tambien como flipped classroom tiene una estrecha relación con las TIC, que actualmente son el medio para continuar dichos procesos educativos. Por esta razón, se presenta en este apartado datos cuantitativos que reflejan el proceso investigativo de los últimos cuatro años.

Figura 2. Distribución de documentos científicos por año

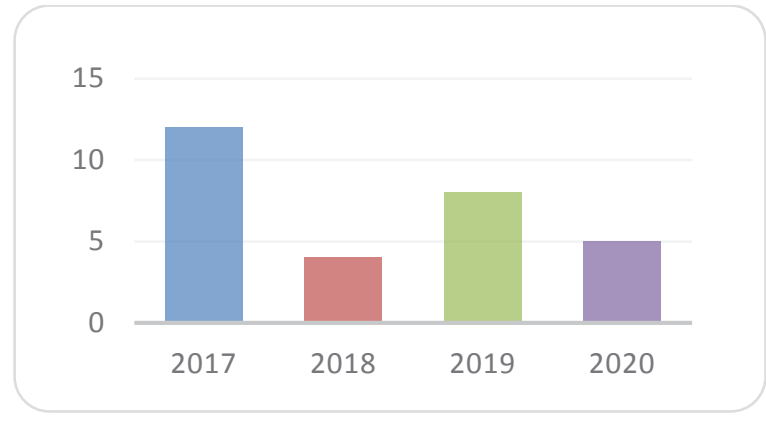

Fuente: elaboración propia

Tomando como referencia los documentos seleccionados, la Figura 2 refleja que en el año 2017 existe un incremento de publicaciones con 12 productos sobre el aula invertida en el Ecuador. Esta tendencia parece atenuarse en el año 2018 con 4 resultados, sin embargo, se retoma en el 2019 con 8 producciones científicas; y, finalmente hasta agosto de 2020, año en curso de la presente investigación, se recopilaron 5 documentos. Al analizar dichos datos, podemos decir que las producciones científicas en el Ecuador han variado en estos últimos 4 años, manteniendo en los años 2017 y 2019 la mayor cantidad de investigaciones En la Tabla 2 se evidencia los autores organizados según el año de publicación de las 29 producciones científicas, en donde se puede apreciar, la mayoría de los estudios se agruparon en el 2017 y 2019, siendo menor en los años 2018 y 2020. 
Tabla 2. Autores clasificados por año de edición

\begin{tabular}{|c|c|c|}
\hline Año & $\mathbf{N}^{\circ}$ & Autores \\
\hline 2017 & 12 & $\begin{array}{l}\text { Montenegro y Solórzano, (2017) ; Zambrano, y Loor (2017); Méjico y Sánchez } \\
\text { (2017); Aguirre (2017); López, (2017) ; Salazar (2017); Escobar (2017); } \\
\text { Godoy y García (2017); León (2017); Mejía et al. (2017); Ortega (2017); } \\
\text { Salazar y Orellana (2017) }\end{array}$ \\
\hline 2018 & 4 & Rivera y García (2018); Tigse (2018); Calderon (2018); Cedeño et al. (2018) \\
\hline 2019 & 8 & $\begin{array}{l}\text { Aycart Carrasco (2019); Rivero-Guerra (2019); Iza (2019); Chicaiza (2019); } \\
\text { Herrera et al. (2019) ; Pesántez (2019) ; Bermeo y Zorrilla (2019); García y } \\
\text { Paredes (2019) }\end{array}$ \\
\hline 2020 & 5 & $\begin{array}{l}\text { Almeida (2020); Arias y Rodríguez (2020); Cobos et al. (2020); Cedeño y } \\
\text { Vigueras (2020); Urbina y Vera (2020) }\end{array}$ \\
\hline
\end{tabular}

Fuente: elaboración propia.

Para la presente investigación se procedió a extraer datos importantes, mediante una lectura de los artículos y tesis con la finalidad de determinar sus aportes a la investigación, asimismo, compararlos de forma cualitativa. A continuación, se muestra la figura 3.

Figura 3. Palabras claves más utilizadas.

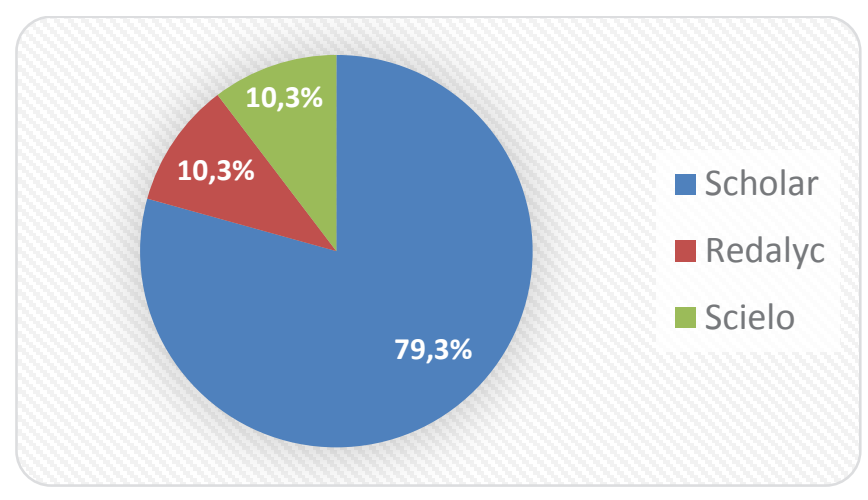

Fuente: elaboración propia.

En la figura 4 se puede visualizar la distribución de 29 producciones científicas con sus respectivos porcentajes de acuerdo a las bases de datos: el buscador académico Google Scholar permitió extraer 27 documentos correspondientes a un 79,3\%, y le sigue Scielo y Redalyc con misma cantidad de documentos, en este caso 3 cada uno, que equivale al 10,3\% individualmente. 
Figura 4. Porcentaje de artículos encontrados por Fuente

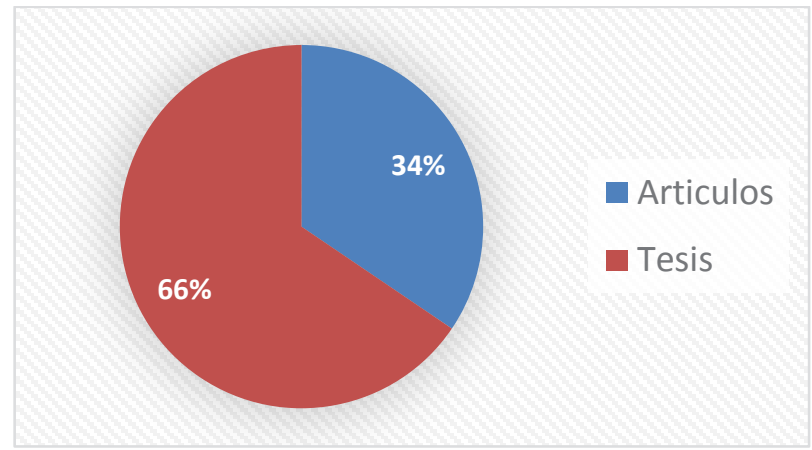

Fuente: elaboración propia.

Como se pudo evidenciar anteriormente, se utilizó diversas fuentes de búsqueda como las bases de datos Redalyc y Scielo, además, el buscador académico Google Scholar. En la Figura 5, observamos la distribución de los 10 artículos investigados que estos corresponden al 34\% y19 tesis que equivale al 66\%. Al analizar, los porcentajes, existe un nivel bajo de socialización académica en publicaciones de artículos con relación a las tesis que presentan trabajos de campo, propuestas y experiencias de aprendizaje.

Figura 5. Tipo de documento.

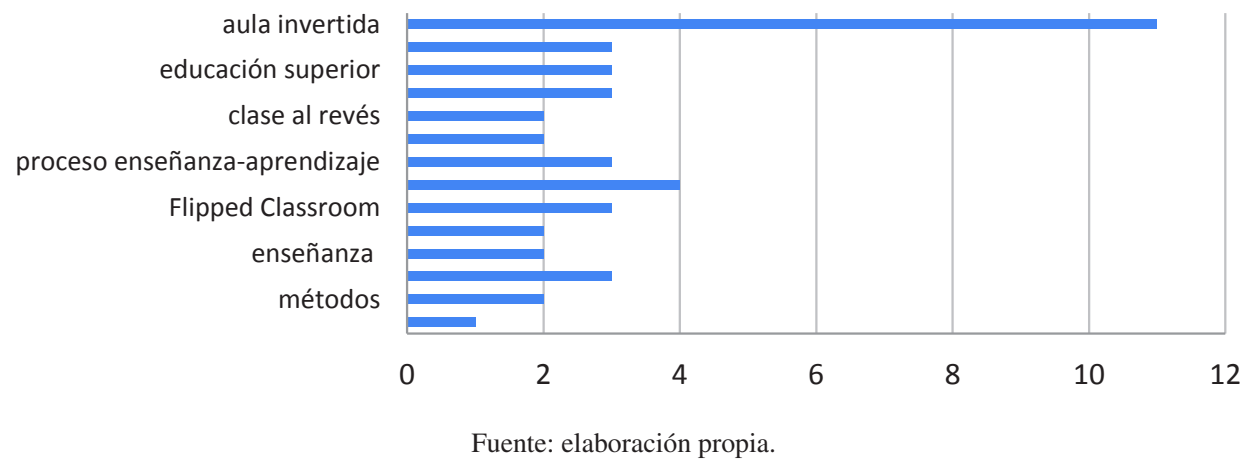

Se identificaron 22 documentos de investigación empírica que corresponden a un 76\%, mientras que los 7 restantes, equivalente al $24 \%$ son estudios de investigación teórica, tal y como se muestra en la Figura 6. 
Figura 6. Tipo de estudio.

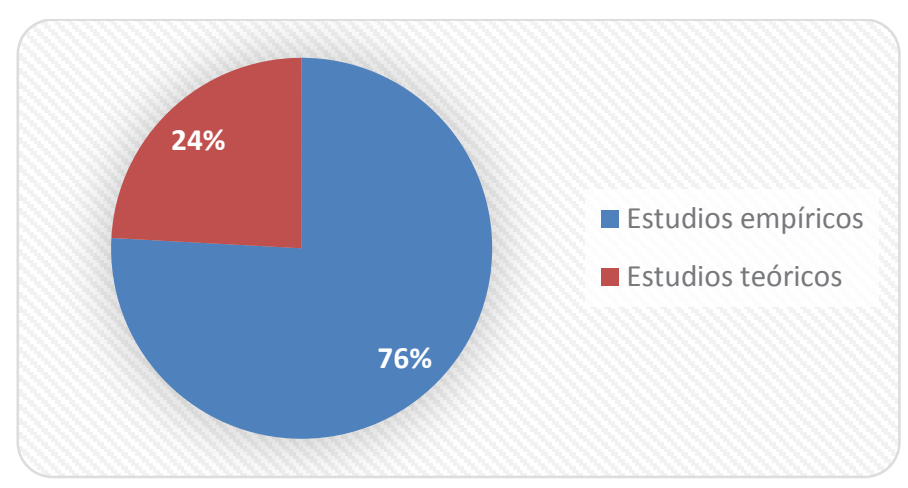

Fuente: elaboración propia.

El aula invertida ha tenido gran acogida en instituciones públicas y privadas, destacándose en especial en los niveles educativos Educación Básica, Bachillerato y los primeros semestres de la Universidad. A partir de las 19 tesis, se evidencia el mayor número de resultados en Bachillerato con 7 resultados entre ellos 2 en primero de Bachillerato, 2 en segundo de Bachillerato y 1 en Bachillerato Internacional. Le sigue con 6 resultados el nivel de Educación General Básica, donde se presentaron en los grados de tercero, quinto, séptimo, sexto y noveno de básica, con 4 resultados se encuentra el nivel de Educación Superior y finalmente se evidencia 2 resultados los cuales fueron aplicados en toda la institución educativa.

Figura 7. Niveles educativos

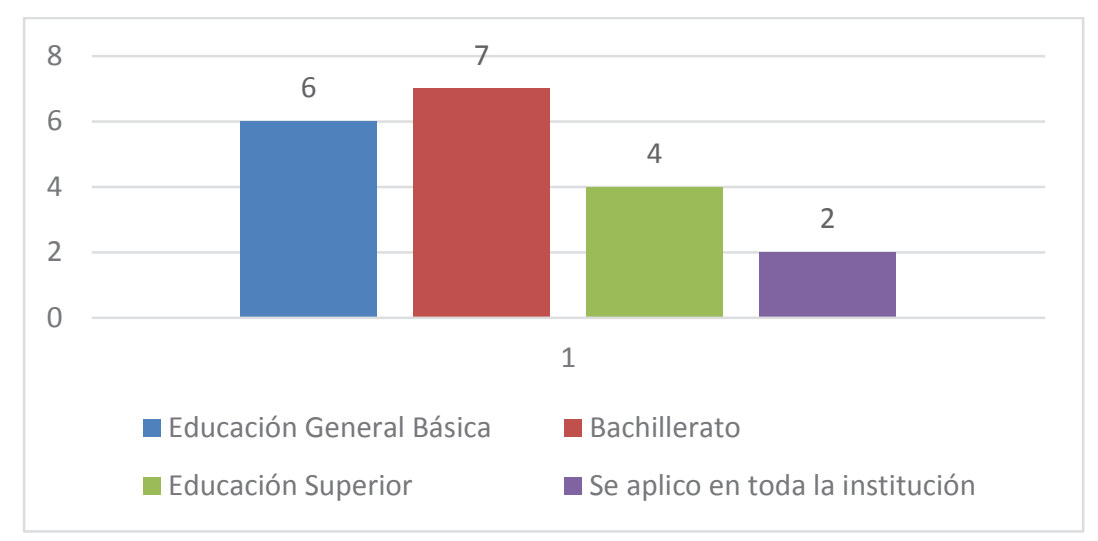

Fuente: elaboración propia. 
Con ello, en la figura 8 se refleja las asignaturas en las que se ha implementado esta metodología, las cuales presentan un $33 \%$ que se aplicó en todas las materias, continua la asignatura del idioma inglés con una apertura del $22 \%$, en la materia de Lengua y Literatura con el 11\%, de igual forma Matemáticas con el 11\%, Ciencias Naturales con el $5 \%$ y las asignaturas de Ciencias Sociales, Botánica y Código Orgánico con 6\% cada una de ellas. A partir de estos resultados se puede evidenciar que la metodología tiene gran acogida en cualquier área del saber, como es el caso del aprendizaje del idioma inglés que muestra un porcentaje significativo, pues al revisar estos documentos se ubicó que las problemáticas que impulsaron la implementación de este modelo, estaban relacionadas con la desmotivación, desinterés y dificultades que presentaban los estudiantes al aprender cada una de las habilidades lingüísticas que se necesitan para aprender un nuevo idioma.

Figura 8. Porcentaje por asignatura.

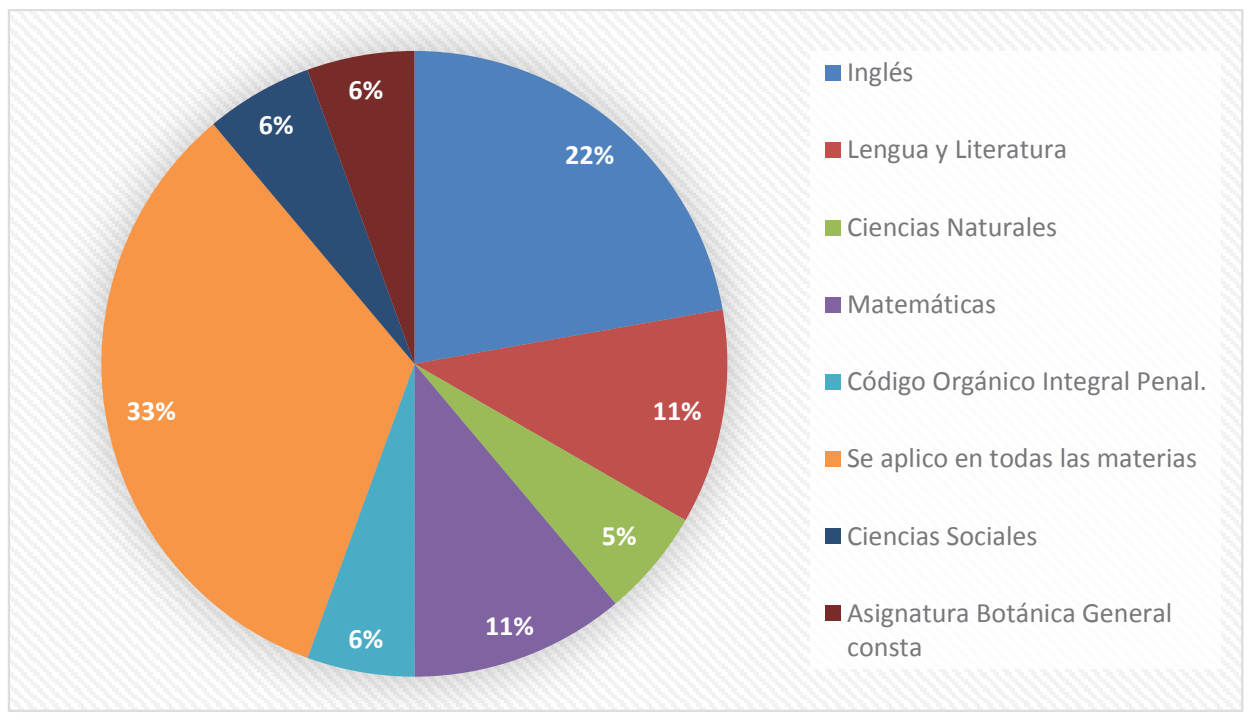

Fuente: elaboración propia.

De acuerdo con la figura 9, las 19 tesis recopiladas muestran que las provincias con mayor flujo son: Pichincha con $42 \%$ que corresponde a 8 tesis, continua Guayaquil con el $21 \%$ lo que equivale a 4 tesis, luego con el porcentaje del 10\% está la provincia de Manabí con 2 tesis, con mismos porcentajes encontramos las provincias de Los Ríos 2, Tungurahua 2 con $11 \%$ y finalmente Cotopaxi que presenta menor flujo del $5 \%$, lo que corresponde a 1 tesis. 
Figura 9. Distribución por provincia.

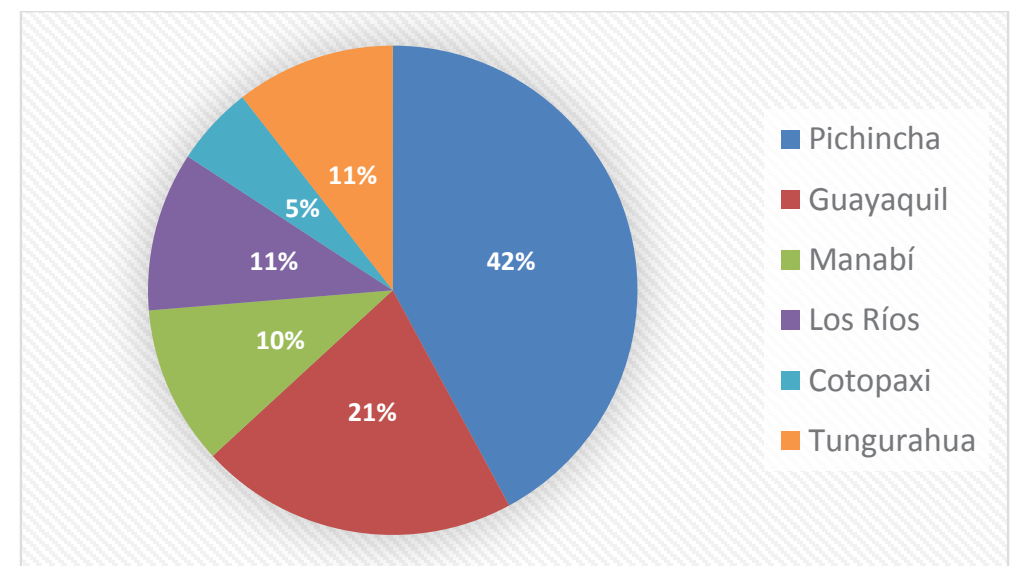

Fuente: elaboración propia.

Para la investigación se procedió a clasificar y organizar los aportes más relevantes de las 19 tesis mediante una matriz que refleja cómo los aportes más relevantes por los autores son: Guías didácticas, recursos educativos, propuestas y estrategias como lo muestra la figura 10 .

Figura 10. Aportes relevantes de las tesis.

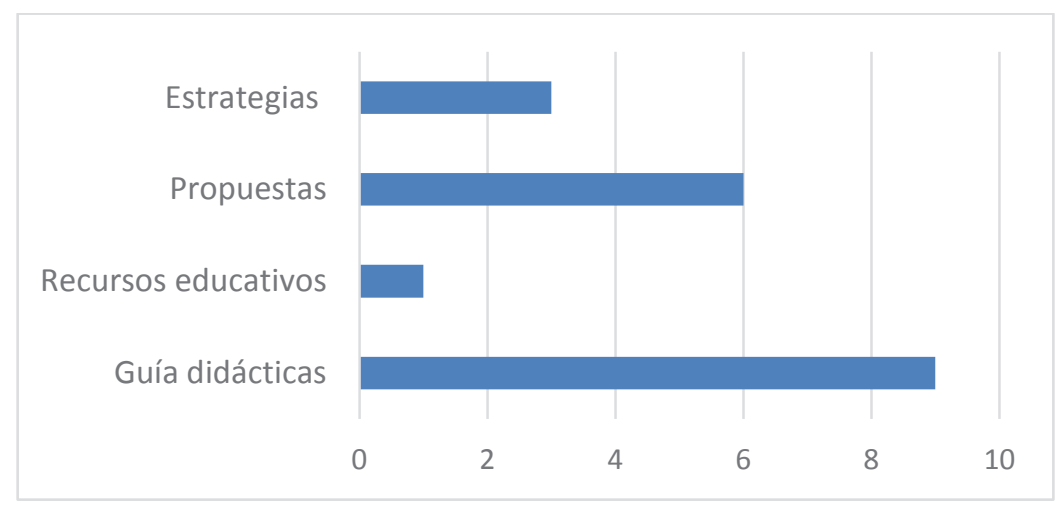

Fuente: elaboración propia. 
A partir de la revisión documental de las producciones empíricas, se arrojan varios resultados de la implementación del aula invertida. Destacando principalmente la motivación y participación activa de los estudiantes durante la clase y como efecto de esto, existen mejoras en el rendimiento escolar. Favoreciendo de esta manera el proceso de enseñanza aprendizaje de los discentes y con ello, la satisfacción de los padres de familia, docentes y autoridades de la institución (Zambrano y Loor, 2017).

En esta línea, el educando muestra compromiso, interés y adquiere nuevas destrezas, mediante actividades divertidas y agradables (Bermeo y Zorrilla, 2019). Estos factores promueven espacios de diálogo, debate, preguntas e investigación antes, durante y después de cada clase. De este modo, al docente se le facilita la planificación de actividades que promuevan la interacción y participación con los estudiantes.

Por otra parte, este modelo de aprendizaje invertido no solo incrementa la motivación del estudiante sino disminuye el porcentaje de deserción escolar (Tigse, 2018). Sin duda, los educandos desarrollan habilidades como el autoaprendizaje, la curiosidad, refuerzan su confianza, conocen antes sobre la temática y resuelven dudas e inquietudes junto al docente.

La metodología flipped classroom da la vuelta a los procesos educativos, planteando actividades previas en casa, lo cual resulta beneficioso para el docente. Debido a que este, puede aprovechar y optimizar el tiempo en el aula, para acciones prácticas, experimentales, interesantes y aquellas que necesitan retroalimentación (Chicaiza, 2019; Almeida, 2020). De esta manera, no solo el estudiante se favorece de esta metodología activa, sino el docente adquiere nuevas estrategias.

Por otra parte, en algunos documentos empíricos se evidencia la relación de esta metodología con los recursos TIC, tanto para el trabajo autónomo como colaborativo. Reflejando resultados positivos en el estudiante, pues este se mantiene activo y dinámico, gracias a los ambientes de aprendizaje interactivos (López, 2017). Sin embargo, si alguno de los sujetos de la educación no posee dichos recursos tecnológicos, se convierte en una limitación para el proceso educativo.

\section{CONCLUSIONES}

De acuerdo con la muestra seleccionada, la metodología del aula invertida, específicamente en el Ecuador, actualmente se encuentra con un índice bajo de aportes científicos. Asimismo, presenta escasas publicaciones en bases de batos científicas como Redalyc y Scielo, y un alto índice en el buscador de Google Académico. Por tanto, el estudio refleja 10 artículos y 19 tesis, lo cual demuestra que dichas tesis no alcanzan el nivel de socialización con la comunidad académica. Por otra parte, de 24 provincias que tiene este país, solo en 6 de ellas, se ha aplicado este método; las tres con mayor incidencia son Pichincha, Guayaquil y Tungurahua. Es importante destacar que en nivel de Educación Inicial existe un vació en el estudio de esta temática; para futuras investigaciones se sugiere realizar estudios y propuestas de la misma.

En definitiva, la implementación del aula invertida en el Ecuador ha tenido grandes resultados que han favorecido tanto en los estudiantes como los docentes. Esta metodología 
permite que desde casa se activen los conocimientos previos de los educandos, generando motivación, interés y curiosidad. Además, todo esto permite que el docente aproveche el espacio en el aula para la participación, el dialogo y las preguntas; mejorando el ambiente de aprendizaje, lo cual beneficia al rendimiento académico y disminuye la deserción escolar.

En síntesis, los datos reflejan que la clase inversa puede ser aplicada en todas las asignaturas y en casi todos los niveles educativos, pues es importante destacar que en nivel de Educación Inicial existe un vació al no evidenciarse estudios de esta temática, por esta razón, para futuras investigaciones se sugiere realizar indagar y profundizar la misma.

Por otra parte, se puede concluir que existen varias propuestas, recomendaciones, experiencias y fundamentos teóricos sobre el flipped classroom en cada una de las tesis y artículos recopilados para el presente estudio. Sin embargo, se destaca en el área del idioma inglés un mayor número de documentos, debido a que en esta asignatura se evidenciaron varias problemáticas que orientaron a la búsqueda de un nuevo método que les permita aprovechar el tiempo en clase para actividades prácticas y de comunicación; y de mismo modo motivar al estudiante para que este sea el protagonista de su proceso de aprendizaje.

\section{REFERENCIAS BIBLIOGRÁFICAS}

Aguirre, L. (2017). El uso del aula invertida en el autoaprendizaje del estudiante. Propuesta: diseño de una guía instruccional [Tesis de pregrado, Universidad de Guayaquil]. Repositorio institucional-Universidad de Guayaquil. https://bit.ly/3oHXz5H

Almeida, E. (2020). Aplicación de la metodología Flipped Classroom en el proceso de enseñanzaaprendizaje del idioma inglés [Tesis de posgrado, Universidad Tecnológica Indoámerica]. Repositorio Institucional - Universidad Tecnológica Indoamérica. https://bit.ly/2HSV53p

Arias, A. y Rodríguez, A. (2020). Revisión de propuestas metodológicas: una taxonomía de agrupación categórica. Alteridad, Revista de Educación, 15(2). https://doi.org/https://doi.org/10.17163/ alt. v15n2.2020.01

Aycart Carrasco, F. (2019). Aprendizaje invertido como un enfoque para la calidad formativa universitaria en Ecuador. Revista Conrado, 15(68), 14-21. https://bit.ly/2HKxu5B

Bermeo, A. y Zorrilla, R. (2019). El aula invertida en el desarrollo de las destrezas en el área de matemática. Diseño de una guía didáctica. [Tesis de pregrado, Universidad de Guayaquil]. Repositorio institucional- Universidad de Guayaquil. https://bit.ly/34JAxUd

Calderón, E. (2018). Aurora en el proceso de enseñanza aprendizaje de la asignatura de Ciencias Naturales del segundo bloque en noveno año de Educación General Básica de la Unidad Educativa Casa de la Cultura Ecuatoriana "Benjamín Carrión” Periodo 2017-2018. [Tesis de pregrado, Universidad Central del Ecuador]. Repositorio institucional-Universidad Central del Ecuador. https://bit.ly/3mCOMBY

Cedeño, L., Alvarez, M., Conto, F. y Jaramillo, L. (2018). Modelo de gestión para los procesos de ingreso a la educación superior: su relación con las garantías de continuidad. Revista Cubana de Educación Superior. https://bit.ly/3jP6sGQ

Cedeño, M. y Vigueras, J. (2020). Aula invertida una estrategia motivadora de enseñanza para estudiantes de Educación General Básica. Revista Científica Dominio de Las Ciencias, 6(3), 878-897. https://bit.ly/31Zd0N7

Chicaiza, M. (2019). Flipped Classroom en el desarrollo gramatical del idioma inglés. [Tesis de pregrado, Universidad Central del Ecuador]. Repositorio institucional- Universidad Central del Ecuador. https://bit.ly/2HRUiAf 
Cobos, J., Simbaña, V. \& Jaramillo, L. (2020). El mobile learning mediado con metodología PACIE para saberes constructivistas. Colección de Filosofía de La Educación, (28), 139-162. https:// doi.org/10.17163/soph.n28.2020.05

Escobar, V. (2017). Las técnicas activas de aprendizaje en el rendimiento académico del área de lengua y literatura de los estudiantes de séptimo grado de educación general básica de la Unidad Educativa Pablo Arturo Suárez del cantón Baños de Agua Santa [Tesis de pregrado, Universidad Técnica de Ambato]. Repositorio institucional- Universidad de Ambato. https://bit. $1 \mathrm{y} / 34 \mathrm{Nctjd}$

García, R. y Paredes, R. (2019). Aprendizaje cooperativo en el desarrollo de la producción escrita del idioma inglés en los estudiantes de nivel avanzado II del Centro de Educación Continua de la Escuela Politécnica Nacional durante el periodo 2019-2019 [Tesis de pregrado, Universidad Central del Ecuador]. Repositorio Institucional- Universidad Central del Ecuador. https://bit.ly/34HYQBK

Godoy, N. y García, N. (2017). La lectura crítica en la calidad del aprendizaje significativo propuesta: taller en base de aula invertida [Tesis de pregrado, Universidad de Guayaquil]. Repositorio institucional-Universidad de Guayaquil. https://bit.ly/2TLz3SV

Herrera, M., Perugachi, J. y Baldeón, P. (2019). Las TIC en el desarrollo de clase inversa: experiencia Unidad Educativa Fiscal San Francisco de Quito. Revista Conrado, 15(70), 248-257. https://bit. ly/3jMsLNq

Iza, M. (2019). La clase inversa y su incidencia en el proceso de aprendizaje interactivo en la asignatura de inglés de la Unidad educativa a distancia Monseñor Alberto Zambrano Palacios del Cantón Pastaza. [Tesis de posgrado, Universidad Técnica de Ambato]. Repositorio Institucional-Universidad Técnica de Ambato. https://bit.ly/32074Ds

Kitchenham, B. (2004). Procedures for performing systematic reviews. Keele University (Vol. 33). https://bit.ly/36170F5

León, D. (2017). Enfoque metodológico del aula invertida como estrategia didáctica en los docentes de la Unidad Educativa "Juan E. Verdesoto" [Tesis de pregrado, Universidad, Universidad Técnica de Babahoyo]. Repositorio institucional- Universidad Técnica de Babahoyo. https://bit. ly/3kNnqqJ

López, B. (2017). El modelo aula invertida en el aprendizaje de los morfemas independientes del inglés a estudiantes de primer nivel de inglés de la UDLA periodo 2016-2017 [Tesis de pregrado, Universidad Central del Ecuador]. Repositorio institucional-Universidad Central del Ecuador. https://bit.ly/2TDwuCC

Mejía, C., Michalón, D., Michalón, R., López, R., Palmero, D. y Sánchez, S. (2017). Espacios de aprendizaje híbridos. Hacia una educación del futuro en la Universidad de Guayaquil. Revista Medisur, 15(3), 350-355. https://bit.ly/2JkLvqX

Méjico, G. y Sánchez, V. (2017). Influencia de la neurosicoeducación en el nivel de desarrollo motriz en los estudiantes de sexto año de Educación General Básica de la Unidad Educativa "Ing. Patricio Espinosa" zona 9, provincia pichincha, cantón quito, parroquia Chilibulo, distrito 6, periodo académico 2014-2015. Diseño de una guía didáctica con enfoque aula invertida. [Tesis de pregrado, Universidad de Guayaquil]. Repositorio institucional-Universidad de Guayaquil. https://bit.ly/3el8eyr

Montenegro, A. y Solórzano, D. (2017). Influencia del déficit de atención en el bajo desempeño escolar en los estudiantes de quinto año de Educación General Básica, Propuesta; diseño de una guía didáctica con enfoque aula invertida [Tesis de pregrado, Universidad de Guayaquil]. Repositorio institucional- Universidad de Guayaquil. https://bit.ly/31UIILk

Okoli, C. \& Schabram, K. (2010). A Guide to Conducting a Systematic Literature Review of Information Systems Research. Working Papers on Information Systems, 10 (2010). https://doi. org/10.2139/ssrn.1954824

Ortega, C. (2017). El uso de las herramientas virtuales y su incidencia en el aprendizaje significativo de los estudiantes del segundo año de Bachillerato de Educación Básica de la Unidad Educativa 
“José Rodríguez Labandera” del cantón Quevedo. [Tesis de pregrado, Universidad Técnica de Babahoyo]. Repositorio institucional-Universidad Técnica de Babahoyo. https://bit.ly/3e6GLAD

Pesántez, M. (2019). Análisis sobre las prácticas inclusivas del profesorado universitario: Estudio de caso de la carrera de Educación Especial de la Universidad Nacional de Educación [Tesis de doctorado, Universidad de Vigo]. Repositorio institucional- Universidad de Vigo. https://bit. ly/34IQYjn

Rivera, F. y García, A. (2018). Aula invertida con tecnologías emergentes en ambientes virtuales en la Universidad Politécnica Salesiana del Ecuador. Revista Cubana de Educación Superior, (1), 108-123. https://bit.ly/2OEWcE3

Rivero-Guerra, A. O. (2019). Impacto de tres modelos de enseñanza de la asignatura Botánica General sobre el rendimiento académico. Revista Formación Universitaria, 12(3), 67-80. https://doi. org/10.4067/S0718-50062019000300067

Salazar, A. y Orellana, A. (2017). Influencia del blog educativo en la calidad del aprendizaje significativo en los y las estudiantes del segundo año de Bachillerato General Unificado en Ciencias, en la asignatura de Matemática de la Unidad Educativa "Océano Pacífico", en la zona 4, Distrito 13D11, Provincia de Manabí, Cantón Sucre, Parroquia Charapotó Período lectivo 2015-2016. Diseño una guía digital educativa con enfoque aula invertida. [Tesis de pregrado, Universidad de Guayaquil]. Repositorio institucional-Universidad de Guayaquil. https://bit.ly/3mxOavI

Salazar, M. (2017). Aula invertida y el proceso de aprendizaje a los estudiantes de la Escuela de Formación de Policías de la subzona Cotopaxi No. 5 en materia de Código Orgánico Integral Penal. [Tesis de pregrado, Universidad Regional Autónoma de los Andes]. Repositorio institucional-Universidad Autónoma de los Andes https://bit.ly/3kOX87o

Tigse, C. (2018). El impacto de la implementación del bachillerato internacional (BI) con el programa del diploma (PD) en búsqueda de la calidad y excelencia de la Unidad Educativa Tumbaco en los años 2017-2018 [Tesis de posgrado, Universidad Andina Simón Bolívar]. Repositorio institucional-Universidad Andina Simón Bolívar -https://bit.ly/37XSssd

Urbina, C. y Vera, P. (2020). Formación E-learning y B-learning en pedagogía didáctica, con apoyo de las TACS para el profesorado de la carrera de ingeniería en sistemas computacionales y software: bases para un plan de formación E-learning y B-learning. [Tesis de Pregrado, Universidad de Guayaquil] Repositorio institucional- Universidad de Guayaquil. https://bit. ly/35UIYvo

Zambrano, M. y Loor, R. (2017). Influencia de técnicas de estudio en la calidad de desempeño escolar en estudiantes de quinto grado de Educación General Básica de la Unidad Educativa "Dr. Luis Aveirga Barberán”, Zona 4, Distrito 5, Provincia Manabí, Cantón El Carmen, Parroquia El Carmen [Tesis de pregrado, Universidad de Guayaquil]. Repositorio institucional-Universidad de Guayaquil. https://bit.ly/326oej8 